\title{
Cluster formation and molecular cloud cores in the Magellanic Clouds
}

\author{
Yasuo Fukui \\ Department of Astrophysics, Nagoya University, Chikusa-ku, Nagoya 464-8602, Japan \\ email: fukui@a.phys.nagoya-u.ac.jp
}

\begin{abstract}
The Magellanic Clouds, consisting of the LMC, the SMC and the Bridge offer an ideal laboratory for studying cluster formation in a lower metal abundance environment at an unrivaled closeness to us among external galaxies. It is known that very young populous clusters like R136 are still being formed in the LMC, where populous clusters include more than 10000 stars tightly gravitationally bound. In this talk, I will present mm and sub-mm CO observations of the Magellanic molecular clouds obtained with NANTEN, SEST, and ASTE at spatial resolutions of 5-50pc. I will then use these $\mathrm{CO}$ data to identify the parent cloud cores for populous clusters and discuss the cluster formation by comparing the cloud properties with those of the Milky Way.
\end{abstract}

Keywords. Magellanic Clouds, GMC, CO, cluster formation

\section{Introduction}

Most massive stars are formed in giant molecular clouds (= "GMCs" hereafter). It is therefore of vital importance to understand star formation processes in GMCs in our continuing efforts to elucidate galaxy evolution and massive star formation. It is to be noted that the spatially resolved GMCs studied so far in terms of star formation are limited to the solar vicinity, within a few kpc of the sun at most, because of heavy contamination and/or obscuration in the galactic disk. In order to better understand GMCs and star formation, we should undoubtedly extend a survey for GMCs into nearby galaxies.

CO observations toward the Local Group galaxies have been mostly limited to the northern sky (e.g., Tacconi \& Young 1987; Wilson \& Scoville 1990; Ohta et al. 1992; Ohta et al. 1993; Wilson 1995; Neininger et al. 1998; Taylor, Kobulnicky, \& Skillman 1998; Loinard et al. 1999), although spatial resolutions and sensitivities high enough to resolve GMCs were achieved only with the largest mm-wave dishes or interferometers for the M31 group at $~ 800 \mathrm{kpc}$ (Neininger et al. 1998; Engargiola et al. 2003). This large distance however hampered to reveal how star formation is taking place in the individual GMCs (e.g., Engargiola et al. 2003 for M33).

The LMC is the nearest neighbour to our own galacy and is actively forming stars as populous clusters (Hodge 1960) or OB associations as well as HII regions. It is the most suitable target for a detailed comprehensive study of GMCs, since it is located at $50 \mathrm{kpc}$ from the Sun, 16 times closer than the M31 group, and is nearly face-on, allowing us to unambiguously identify young objects associated with GMCs. The Magellanic Clouds have different environments from the Milky Way. First, the metallicity is significantly lower than in the Milky Way $\left(\mathrm{LMC} \sim 1 / 4 \mathrm{Z}_{\odot}, \mathrm{SMC} \sim 1 / 10 \mathrm{Z}_{\odot}\right.$ Dufour 1984). It is also known that the gas to dust ratio is ten times higher than in the Milky Way (Koornneef 1982). The UV radiation field is an order of magnitude higher, perhaps due to more 


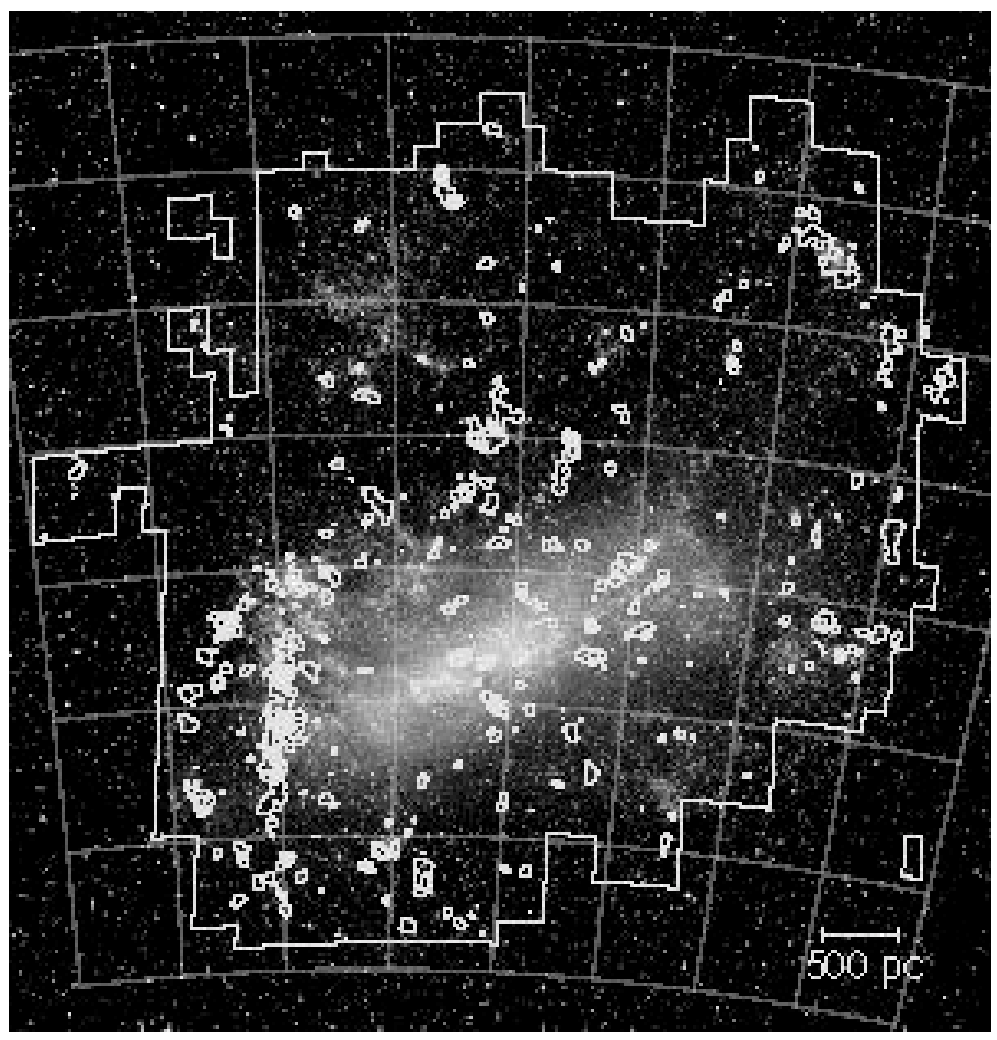

Figure 1. CO $(J=1-0)$ velocity-integrated intensity map of the LMC superposed on an optical image. The contour levels begin at $1.2 \mathrm{~K} \mathrm{~km} \mathrm{~s}^{-1}(=3 \sigma$ noise level $)$, and increase in steps of $2.4 \mathrm{~K} \mathrm{~km} \mathrm{~s}^{-1}$. The broken lines indicate the observed area.

numeorus O stars; for instance, 30 Doradus includes 39 O3 stars (Massey \& Hunter 1998).

SEST was used to make extensive observations of CO in the Magellanic Clouds, and several \% of the galaxy was mapped for GMCs (e.g., Johansson et al. 1998). Later, significant progress made by a CO $J=1-0$ survey covering fully the Large Magellanic Cloud (=LMC) made with a $4 \mathrm{~m} \mathrm{~mm} / \mathrm{sub}-\mathrm{mm}$ wave telescope NANTEN. This has provided an opportunity to study a flux-limited sample of 55 GMCs at a spatial resolution of $40 \mathrm{pc}$, below the typical size of a GMC, 100 pc (Fukui et al. 1999, Paper I hereafter; Mizuno et al. 2001b). The CO survey in Paper I has a sensitivity equivalent to molecular column density of $2.7 \times 10^{21} \mathrm{~cm}^{-2}$ where an $X$ factor, $9 \times 10^{20} \mathrm{~cm}^{-2}\left[\mathrm{~K} \mathrm{~km} \mathrm{~s}^{-1}\right]^{-1}$, is adopted. We should note that this relatively high column density limit may lead to underestimates of the cloud size and mass. It is therefore desirable to improve the sensitivity of $\mathrm{CO}$ observations to a level equivalent to molecular column densities as low as $\sim 10^{21} \mathrm{~cm}^{-2}$, similar to the studies of Galactic GMCs (Dame, Hartmann, \& Thaddeus 2001).

\section{Star formation in the LMC}

\subsection{New CO Survey of the $L M C$}

We have performed a new CO survey of the LMC more sensitive than that in Paper I. In this contribution, I present and discuss these new $\mathrm{CO}$ results and their implications on the evolution of GMCs and massive star formation therein. 
$\mathrm{i}[0 \mathrm{pt}] 80 \mathrm{~mm}$

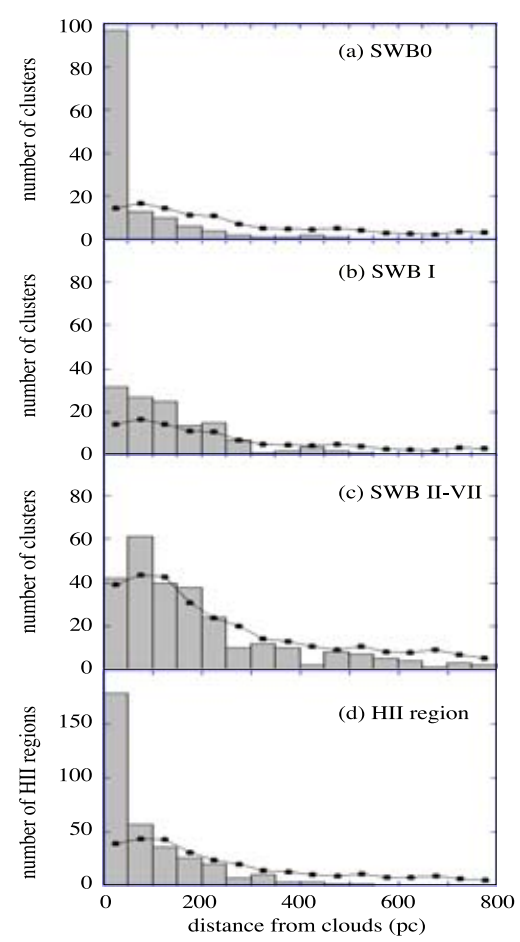

Figure 2. Histograms of the projected distance of the clusters with ages of (a) $\tau<10 \mathrm{Myr}$ (SWB 0), (b) $10 \mathrm{Myr}<\tau<30$ Myr (SWB I), and (c) $30 \mathrm{Myr}<\tau$ (SWB II to SWB VII) (Bica et al. 1996) to the nearest CO emission. The bottom panel (d) shows the projected distances of the H II regions (Davies et al. 1976) to the GMCs. The lines represent the frequency distribution of the clusters or H II regions expected if the same number of objects were distributed at random.

The new survey in the $2.6 \mathrm{~mm} J=1-0$ rotational emission of CO has used NANTEN, a $4 \mathrm{~m} \mathrm{~mm} / \mathrm{sub}-\mathrm{mm}$ wave telescope located in Las Campanas, Chile, and has achieved a sensitivity a factor of $\sim 3$ better than that in Paper I, equivalent to $N\left(\mathrm{H}_{2}\right) \sim 1.0 \times$ $10^{21} \mathrm{~cm}^{-2}$ (see Fukui et al. 2001 and Yamaguchi et al. 2001a for more details of observations). It covered the whole extent of the LMC and has revealed distributions of 270 GMCs with 27,000 observed positions (Figure 1). We have chosen 146 GMCs among the 259, for which reliable estimates of physical quantities are available. The masses of the 146 GMCs range from $4 \times 10^{4} M_{\odot}$ to $4 \times 10^{6} M_{\odot}$ (Fukui et al. 2001).

\subsection{Association with Young Objects}

We have used the present sample of 146 GMCs to learn how star formation is taking place by comparing the GMC distributions with optical signposts of star formation including young stellar clusters (Bica et al. 1996) and HII regions (Davies, Elliott, \& Meaburn 1976, Kennicutt \& Hodge 1986). We note that the detection limit of H II regions is quite low, $L(\mathrm{H} \alpha) \sim 2 \times 10^{36} \mathrm{ergs} \mathrm{s}^{-1}$, corresponding to one fourth of that of Orion Nebula. This allows reliable assignments of the optical signposts to individual GMCs as already demonstrated in Paper I and by Yamaguchi et al. (2001b).

Figure 2 shows the distributions of the projected separations of H II regions and stellar clusters from the nearest GMC. This clearly indicates that the youngest clusters of an age smaller than 10 Myrs, i.e., SWB0 (Bica et al. 1996), and the H II regions are sharply peaked within $100 \mathrm{pc}$ of GMC exhibiting strong spatial correlations. This provides strong 

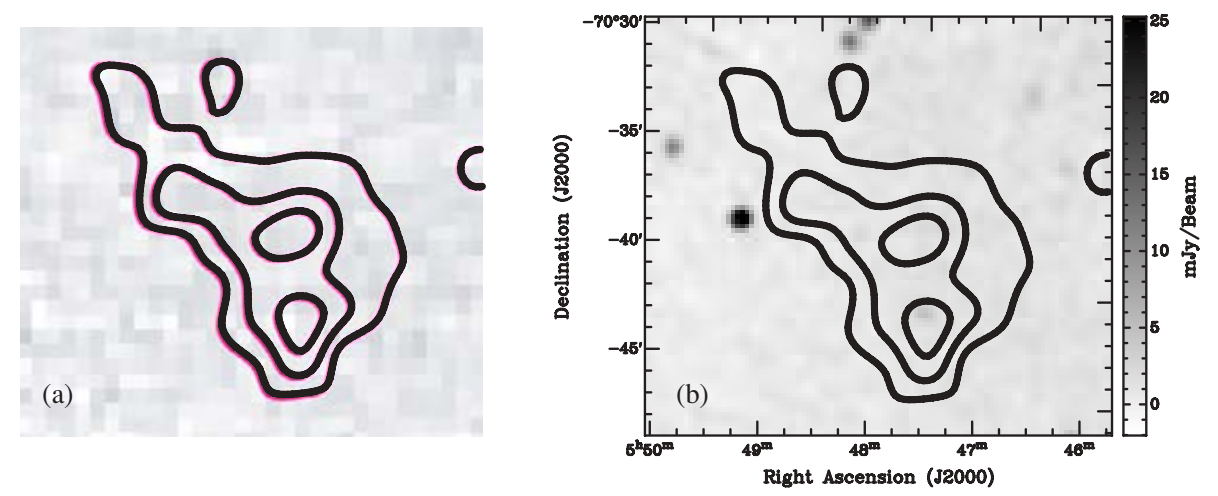

Figure 3. One of the starless GMCs $\left(M_{\mathrm{CO}} \sim 1 \times 10^{6} M_{\odot}\right)$ superposed on (a) $\mathrm{H} \alpha$ image from Kim et al. (1999) and (b) $1.4 \mathrm{GHz}$ continuum image (Filipovic et al. in private communication). The contours are $1.2,2.4,3.6 \mathrm{Kkm} / \mathrm{s}$ showing CO integrated intensity by NANTEN

evidence for the GMCs as natal clouds of these young objects. On the other hand, the older clusters, $\operatorname{SWB1}(\tau>10 \mathrm{Myr})$ or later, show much weaker or no correlation, as is consistent with rapid cloud dissipation after cluster formation (Paper I).

It was shown in Paper I that the GMCs can be classified into three groups based on a sample of 55 GMCs whose masses range from $2 \times 10^{5} M_{\odot}$ to $\left.3 \times 10^{6} M_{\odot} ; 1\right)$ starless GMCs, 2) those with small $\mathrm{HII}$ regions whose $\mathrm{H} \alpha$ luminosity is less than $10^{37} \mathrm{erg} \mathrm{s}^{-1}$, and 3) those with stellar clusters and large $\mathrm{H}$ II regions of $\mathrm{H} \alpha$ luminosity greater than $10^{37}$ erg $\mathrm{s}^{-1}$. We find that the present results confirm the validity of this classification. We have also confirmed that there is no significant difference in physical parameters including mass, CO linewidth, and size among the three groups, as is consistent with Paper I.

In order to confirm that there are no obscured H II regions towards the GMCs, we have further used the most recent Parkes/ATNF radio continuum survey carried out at five frequencies 1.4, 2.45, 4.75, 4.8, and 8.55GHz (Filipovic et al. 1995, 1998). The typical sensitivity limits of these new datasets are quite low, allowing us to reach flux limits equivalent to that in $\mathrm{H} \alpha$. The radio continuum results are summarized in a catalog of 483 sources, and the spectral information allows us to eliminate background sources not related the LMC. By comparing these data with the NANTEN GMCs, we have found that most of the starless GMCs have no radio H II regions, and that only one of them is associated with an H II region detected at radio wavelengths. Figure 3 shows an example of a GMC which has no associated $\mathrm{HII}$ region in radio or $\mathrm{H} \alpha$ emission.

Table 1 summarizes the results of the present comparison between GMCs and young objects, SWB0 clusters and the HII regions including radio sources. It shows that $32 \%$ of the GMCs are starless in the sense that they are not associated with H II regions or youngest clusters. I should note that "starless" here indicates no associated early O star capable of ionizing H II regions, and that it does not exclude the possibility of associated young stars later than B-type not detectable through HII regions. This is the best we can do with the current sensitivity limits at various wavelengths in $\mathrm{CO}$ and optical/radio emissions for spatially resolved extragalactic GMCs; if we were to use GMC samples in galaxies of the M31 group, our sensitivity limits would become higher by a factor of $\sim 300$, implying that one would not be able to discern even early O stars associated with GMCs. 
Table 1. Association of GMCs with young clusters/associations and H II regions

\begin{tabular}{lcccc}
\hline Association & \multicolumn{4}{c}{ Number of GMCs (ratio \%) } \\
& LMC & \multicolumn{3}{c}{ SMC $^{\dagger}$} \\
\hline No association & 46 & $(32 \%)$ & 13 & $(50 \%)$ \\
Only with H II regions & 66 & $(45 \%)$ & & \\
With H II regions and clusters & 34 & $(23 \%)$ & 13 & $(50 \%)$ \\
\hline Total & 146 & 26 & \\
\hline$\dagger$ Mizuno et al. 2001a & \multicolumn{5}{l}{}
\end{tabular}

\subsection{Comparison with the Milky Way}

In the Milky Way, almost all the GMCs in the solar neighborhood, within $\sim 2 \mathrm{kpc}$, are known to be forming massive stars (Blitz 1993). This suggests that any visual extinction in a GMC is not able to veil HII regions or young stars. The reason for this is rather obvious; first, the HII regions create a cavity into the natal GMCs, a well known phenomenon referred to as "Champagne model" (e.g., Tenorio-Tagle 1979). Second, GMCs are highly filamentary as seen in Orion A and others (e.g., Bally et al. 1987; Nagahama et al. 1998) and such a distribution favors disclosing H II regions toward any direction.

A similar situation likely holds in the LMC, 1)because the GMCs in the LMC also show filamentary distributions at higher resolutions (e.g., Israel et al. 2003), and 2)because cavities should be formed more easily due to the less UV extinction (Koornneef 1982) combined with higher UV intensity (Israel et al. 1986) in the LMC than in the Milky Way. We conclude that the starless GMCs are not an artifact of observations and that a significant portion of the GMCs, $\sim 32 \%$, in the LMC are not forming O stars.

\section{Massive and Dense Cores; Candidates for Proto-Cluster Cores}

We have used the ASTE sub-mm 10m telescope to map the selected GMCs in the LMC. The CO $J=3-2$ emission at $345 \mathrm{GHz}$ was used in the observations and one of the $\mathrm{CO}$ distributions is shown in Figure 4 for the region N159. Figure 4 clearly demonstrates the denser CO 3-2 clumps within the GMC traced by the $\mathrm{CO} J=1-0$ emission. We have estimated the clump mass as several times $10^{4} M_{\odot}$ to a few times $10^{5} M_{\odot}$ by using the virial theorem, $M_{\mathrm{vir}}=200 \Delta v[\mathrm{~km} / \mathrm{s}]^{2} R[\mathrm{pc}] M_{\odot}$, where $R$ is the geometrical mean measured at the half intensity contour level. The beam size of 22 arc sec corresponds to $5 \mathrm{pc}$, and the typical clump size here is several pc. The densities of these clumps are roughly estimated to be $\gtrsim 10^{4} \mathrm{~cm}^{-3}$ from the excitation conditions and also from the average of the virial mass. These masses and sizes are nearly similar to those of the populous stellar clusters. I therefore suggest that the CO 3-2 clumps are promising candidates for the precursors to populous clusters. It will be extremely interesting to investigate whether even denser gas exits in these massive clumps, which will be soon undertaken with NANTEN2 sub-mm telescope at higher frequencies. It is also an obvious target for the ALMA to look for detailed structures within these clumps which may tell us more about the individual young massive protostars.

\section{Discussion}

The completeness of the present GMC sample covering the whole LMC enables us to infer the evolutionary timescales of the GMCs. Figure 5 gives a summary of the typical timescales of the four stages of GMC evolution obtained from the present comparison, 


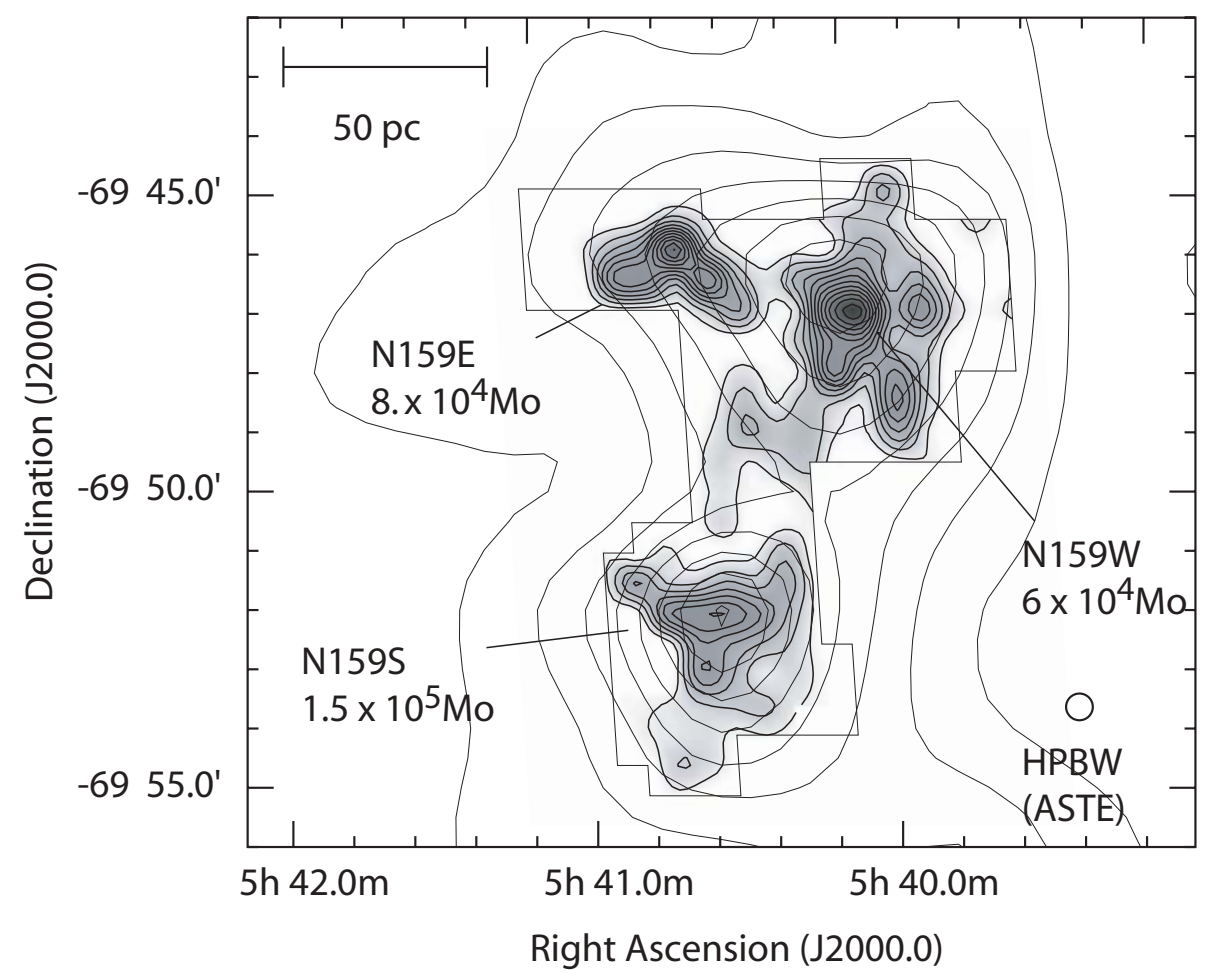

Figure 4. Dense cores in the N159 GMC observed in ${ }^{12} \mathrm{CO}(3-2)$ by ASTE. The image with thick contours indicates the integrated intensity. The contours are from $2 \mathrm{~K} \mathrm{~km} / \mathrm{s}$ with $3 \mathrm{~K} \mathrm{~km} / \mathrm{s}$ intervals. Thin contours show the CO integrated intensity by NANTEN; the lowest contours and the intervals are $1.2 \mathrm{Kkm} / \mathrm{s}$, respectively.

where a steady state evolution is assumed. The 1st stage corresponds to starless GMCs, having a long timecscale of 7 Myrs. This is followed by a phase with small H II regions, implying the formation of a few to several $\mathrm{O}$ stars. The suesequent phase indicates the most active formation of rich clusters including many early $\mathrm{O}$ stars (one of such an example is $\mathrm{N} 159 \mathrm{~N}$ ). In the final phase, the GMC has been more or less dissipated under the strong ionization and stellar winds from $\mathrm{O}$ stars. The total lifetime of a GMC is then estimated as $\sim 20$ Myrs.

We shall discuss the implications of the fact that more than $30 \%$ of the GMCs in the LMC are starless. Blitz (1993) gives a conservative estimate of the time scale of starless GMCs as $\lesssim 3$ Myrs in the Milky Way, since less than $10 \%$ of the GMCs in the solar vicinity are starless. An idea that immediately occurs to explain starless GMCs is that the timescale of star formation and/or cloud contraction is significantly longer in the LMC than in the Milky Way, if we take the difference in time scale to be significant. We propose here that a higher ionization degree in GMCs is the primary cause of the retarded star formation in the LMC.

The ionization degree in a molecular cloud is likely determined by the far-ultra violet (FUV) photons of stellar radiation fields (McKee 1989; Nozawa et al. 1991). In the LMC, the FUV flux is several times higher (Israel et al. 1986) and the dust extinction is smaller by a factor of 3-4 for a given gaseous column density (Koornneef 1982). Thus, the ionization degree in molecular gas should become roughly ten times higher. Since the time scale of the diffusion of magnetic field is proportional to the ionization degree (Spitzer 1978), it seems likely that cloud contraction is effectively retarded by magnetic fields. In 

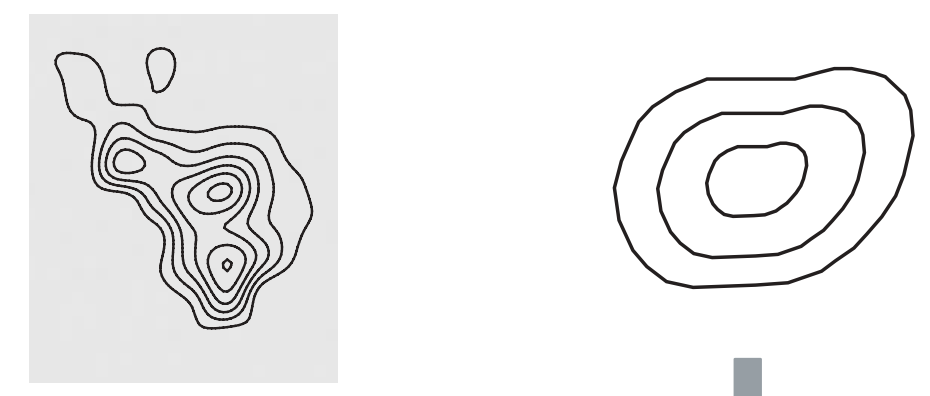

Starless GMC 46 clouds (32\%) 7 Myr
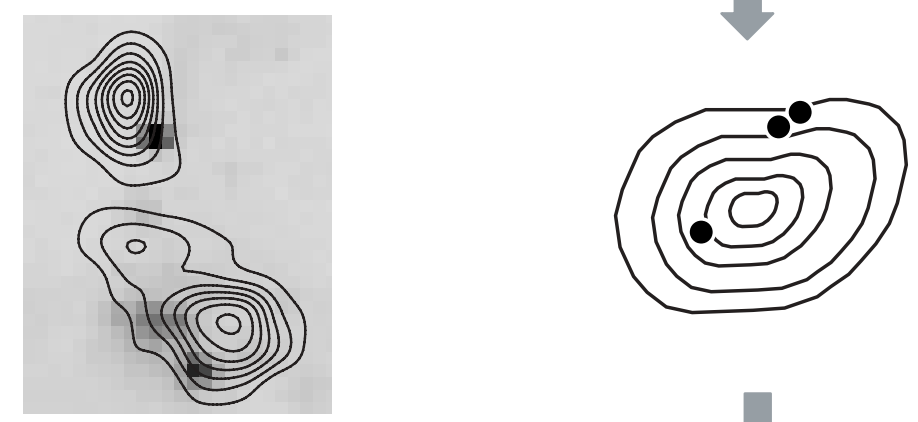

Only HII regions 66 clouds $(45 \%)$ 10 Myr
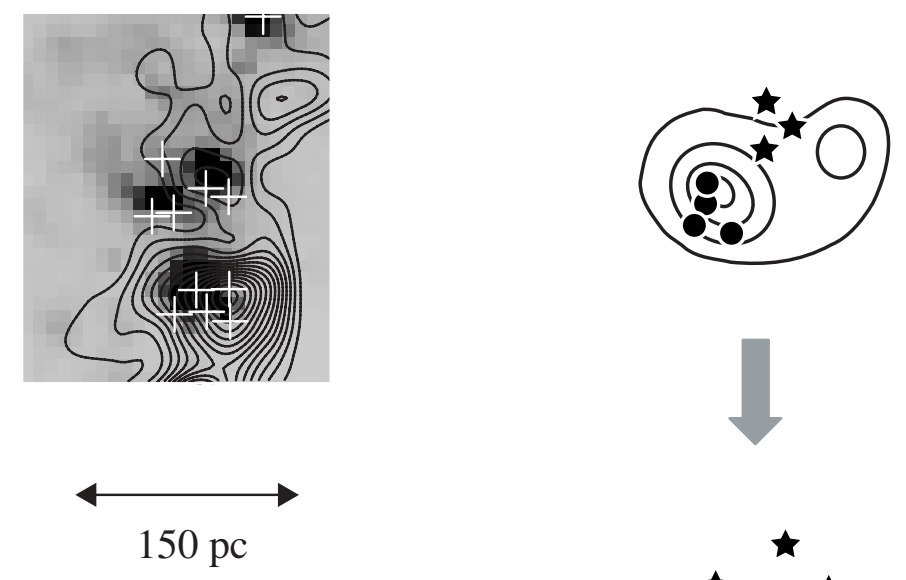

Clusters and HII regions 34 clouds $(23 \%)$ $\sim 5 \mathrm{Myr}$

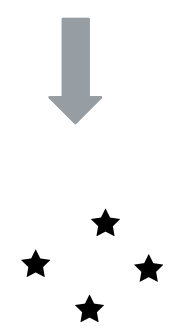

Only clusters

5 Myr

Figure 5. Evolutionary sequence of the GMCs. The left panels are examples of GMCs without any evidence for massive star or cluster formation, with only H II regions, and with H II regions and clusters. The images are $\mathrm{H} \alpha$ from Kim et al. (1999). The contours show the integrated intensity of ${ }^{12} \mathrm{CO}(1-0)$ by NANTEN; both the lowest contour and the intervals are $1.2 \mathrm{~K}$ $\mathrm{km} / \mathrm{m}$. White crosses indicate the positions of SWB 0 clusters (Bica et al. 1996) The right panels illustrate the evolutionary stages; the filled circles and stars represent H II regions and young clusters, respectively.

addition, the cooling rate via molecular and dust emission is likely smaller by a factor of 3-4 in the LMC than in the Milky Way, further helping to slow down star formation. A higher ionization degree and smaller cooling rate are basically the consequences of the 
lower metallicity in the LMC (Dufour 1984) and are likely responsible for the retarded star formation.

We may be able to test this hypothesis by observing GMCs in other galaxies with different metallicities to see if the timescale of massive star formation is dependent on metalicity. At the moment, one of the testable galaxies is the SMC which has a metalicity ten times lower than the Milky Way (e.g., Dufour 1984). This leads us to suggest that there may be more starless GMCs in the SMC. An obstacle to such a test is that the total star formation rate is much lower than LMC, making the statistics poorer in the SMC. Another possibility may be M 33 whose metalicity is similar to the Milky Way. In this spiral galaxy star forming GMCs seem dominant $(\geqslant 75 \%)$, while the existence of starless GMCs is not yet confirmed (Engargiola et al. 2003). We need to wait for new instruments with even higher resolution at $\mathrm{mm} / \mathrm{sub}-\mathrm{mm}$ wavelengths such as ALMA which will allow us to reach resolved GMCs in more distant galaxies.

An alternative idea to explain starless GMCs is that the GMCs in the LMC are of very recent formation, a situation possibly similar to the Maddalena's clouds. It is well known that the LMC has a number of supershells expanding and accumulating interstellar matter (Meaburn 1980; Oey 1996; Kim et al. 1999). Yamaguchi et al. (2001a) investigated the possible correlation between GMCs and supergiant shells and conclude that one third of GMCs may be located towards the shell boundaries, suggesting that a significant number of GMCs may have been formed under triggering by expanding shells. The spatial distribution of the starless GMCs is however fairly random, showing little correlation with supergiant shells.

Finally, we shall comment on the possible link between starless GMCs and the populous stellar clusters, mini-globulars including 10000 stars (Hodge 1960); detailed plots of the number of stars in clusters are found in Kumai, Basu, \& Fujimoto (1993) and Hunter et al. (2003). It is tempting to speculate that there is a link between populous clusters and the starless GMCs because GMCs are formation sites of populous clusters (Paper I). A possible explanation is that the longer timescale of star formation allows the formation of proto-cluster molecular condensations as massive as $10^{5} M_{\odot}$, which can lead to form the rich populous clusters. This may seldom happen in the Milky Way because of the rapid star formation immediately after formation of proto-cluster molecular condensations referred to as "hot cores" having mass of only $\sim 10^{3} M_{\odot}$ (e.g., Shirley et al. 2003). Details of massive, dense proto-cluster molecular condensations of $10^{5} M_{\odot}$ should be obtained with ALMA within several years.

\section{Acknowledgements}

The NANTEN project is based on a mutual agreement between Nagoya University and the Carnegie Institution of Washington (CIW). We greatly appreciate the hospitality of all the staff members of the Las Campanas Observatory of CIW. We are thankful to many Japanese public donors and companies who contributed to the realization of the project. This work is based on a collaboration with A. Kawamura, T. Onishi, N. Mizuno, T. Minamidani, Y. Mizuno, and A. Mizuno. I would like to acknowledge Drs. L. SteavelySmith and M. Filipovic for the kind use of their radio continuum data prior to publication and Dr. L. Johansson for the collaboration on SEST. This work is financially supported in part by a Grant-in-Aid for Scientific Research from the Ministry of Education, Culture, Sports, Science and Technology of Japan (No. 15071203) and from JSPS (No. 14102003).

\section{References}

Bally, J., Langer, W. D., Stark, A. A., \& Wilson, R. W. 1987, ApJ, 312, L45 
Bica, E., Claria, J. J., Dottori, H., Santos, J. F. C. Jr., \& Piatti, A. E. 1996, ApJS, 102, 57

Blitz, L. 1993, Nature, 364, 757

Davies, R. D., Elliott, K. H., \& Meaburn, J. 1976, MNRAS, 81, 89

Dufour, R. J. 1984, in Structure and Evolution of the Magellanic Clouds, ed. S. van den Bergh \& K. S. de Boer (Dordrecht: Reidel Publishing Co.) 353

Engargiola, G., Plambeck, R. L, Rosolowsky, E., \& Blitz, L. 2003 ApJS, 149, 343

Filipovic, M., D., Haynes, R. F., White, G. L., Jones, P. A., Klein, U., \& Wielebinski, R. 1995, $A A S, 111,311$

Filipovic, M. D., Jones, P. A., White, G. L., \& Haynes, R. F. 1998, AAS, 130, 441

Fukui, Y., Mizuno, N., Yamaguchi, R., Mizuno, A., Onishi, T., Ogawa, H., Yonekura, Y., Kawamura, A., Tachihara, K., Xiao, K., et al. 1999, PASJ, 51, 745 (Paper I)

Fukui, Y., Mizuno, N., Yamaguchi, R., Mizuno, A., \& Onishi, T. 2001, PASJ, 53L, 41 Hodge, P. 1960, ApJ, 131, 351

Hunter, D. A., Elmegreen, B. G., Dupuy, T. J., \& Mortonson, M. 2003, AJ, 126, 1836

Israel, F. P., de Graauw, Th., van de Stadt, H., \& de Vries, C. P. 1986, ApJ, 303, 186

Israel, F. P., Johhansson, L. E. B, Rubio, M., Garay, G., de Graauw, Th., Booth, R. S., Boulanger, F., Kutner, M. L., Lequeux, J., \& Nyman, L.-A. 2003, A $\& A, 4$ 406, 817

Johansson, L. E. B., Greve, A., Booth, R. S., Boulanger, F., Garay, G., Graauw, Th. de, Israel, F. P., Kutner, M. L., Lequeux, J., Murphy, D. C., Nyman, L.-A., \& Rubio, M. 1998, A\&̈A, 331,857

Kennicutt, R. C., Jr., \& Hodge, P. W. 1986, ApJ, 306, 130

Kim, S., Dopita, M. A., Staveley-Smith, L., \& Bessel, M. S. 1999, AJ, 118, 2797

Koornneef, J. 1982, A\& A, 107, 247

Kumai, Y. Basu, B., \& Fujimoto, M. 1993, ApJ, 404, 144

Loinard, L., Dame, T., Heyer, M. H., Lequeux, J., \& Thaddeus, P. 1999, A\&A, 351, 1087

Massey, D., \& Hunter, D. A. 1998, ApJ, 493, 180

McKee, C. F. 1989, ApJ, 345, 782

Meaburn, J. 1980, MNRAS, 192, 365

Mizuno, N., Rubio, M., Mizuno, A., Yamaguchi, R., Onishi, T., \& Fukui, Y. 2001a, PASJ, 53, L45

Mizuno, N., Yamaguchi, R., Mizuno, A., Rubio, M., Abe, R., Saito, H., Onishi, T., Yonekura, Y., Yamaguchi, N., Ogawa, H., \& Fukui, Y. 2001b, PASJ, 53, 971

Nagahama, T., Mizuno, A., Ogawa, H., \& Fukui, Y. 1998, AJ, 116, 336

Neininger, N., Guelin, M., Ungerechts, H., Lucas, R., \& Wieleninski, R. 1998, Nature, 395, 871

Nozawa, G., Mizuno, A., Teshima, Y., Ogawa, H., \& Fukui, Y. 1991, ApJS, 77, 647

Oey, M. S. 1996, ApJ, 467, 666

Ohta, K., Sasaki, M., Yamada, T., Saito, M., \& Nakai, N. 1992, PASJ, 44, 585

Ohta, K., Tomita, A., Saito, M., Sasaki, M., \& Nakai, N. 1993, PASJ, 45L, 21

Shirley, Y. L., Evans, N. J. II, Young, K. E., Knez, C., \& Jaffe, D. T. 20 03, ApJS, 149, 375

Spitzer, L. Jr.1978, Physical Processes in the Interstellar Medium (New York: Wiley Interscience)

Tacconi, L. J., \& Young, J. S. 1987, ApJ, 322, 681

Taylor, C. L., Kobulnicky, H., A., \& Slillman, E. D. 1998, AJ, 116, 2746

Tenorio-Tagle, G. 1979, A\&A, 71, 59

Wilson, C. D. 1995, ApJ, 448, L97

Wilson, C. D., \& Scoville, N. 1990, ApJ, 363, 435

Yamaguchi, R., Mizuno, N., Onishi, T., Mizuno, A., \& Fukui, Y. 2001a, PASJ, 53, 959

Yamaguchi, R., Mizuno, N., Mizuno, A., Rubio, M., Abe, R.,Saito, H., Moriguchi, Y., Matsunaga, L., Onishi, T., Yonekura, Y., \& Fukui, Y. 2001b, PASJ, 53, 985 